\title{
Highly sensitive and miniature microfiber-based ultrasound sensor for photoacoustic tomography
}

\author{
Liuyang Yang ${ }^{1}$, Yanpeng Li ${ }^{1}$, Fang Fang ${ }^{1}$, Liangye $\mathrm{Li}^{1}$, Zhijun Yan ${ }^{1}$, \\ Lin Zhang² and Qizhen Sun®1*
}

\begin{abstract}
A microfiber with large evanescent field encapsulated in PDMS is proposed and demonstrated for ultrasound sensing. The compact size and large evanescent field of microfiber provide an excellent platform for the interaction between optical signal and ultrasound wave, exhibiting a high sensitivity of $3.5 \mathrm{mV} / \mathrm{kPa}$, which is approximately 10 times higher than the single-mode fiber sensor. Meanwhile, a phase feedback stabilization module is introduced into the coherent demodulation system for long-term stable measurement. In addition, a photoacoustic tomography experiment with the microfiber ultrasound sensor is implemented to verify the excellent performance on imaging, with the depth of $12 \mathrm{~mm}$, the highest lateral resolution of $65 \mu \mathrm{m}$ and axial resolution of $250 \mu \mathrm{m}$, respectively. The highly sensitive microfiber ultrasound sensor provides a competitive alternative for various applications, such as industrial non-destructive testing, biomedical ultrasound and photoacoustic imaging.
\end{abstract}

Keywords: ultrasound sensor; microfiber; photoacoustic tomography

Yang LY, Li YP, Fang F, Li LY, Yan ZJ et al. Highly sensitive and miniature microfiber-based ultrasound sensor for photoacoustic tomography. Opto-Electron Adv 5, 200076 (2022).

\section{Introduction}

Ultrasound sensors play an important role in many fields, such as biomedical ultrasound imaging ${ }^{1-3}$, nondestructive testing (NDT) ${ }^{4,5}$, partial discharge detection $^{6,7}$ and structural health monitoring ${ }^{8}$. The traditional ultrasound sensors are mainly based on piezoelectric effect, which suffer from several limitations ${ }^{9,10}$. Firstly, the sensitivity of piezoelectric sensors depends on the size of piezoelectric elements, resulting in relatively large size of sensors. Meanwhile, the piezoelectric ultrasonic sensors usually with a narrow response bandwidth, and thereby prevent a faithful representation of the incident acoustic wave. In addition, the piezoelectric sensors are susceptible to electromagnetic interference (EMI), and therefore fail to work in extreme environments with strong electromagnetic. Therefore, the development of highly sensitive, broad bandwidth and cost-effective ultrasound sensors is highly demanded in practical applications.

Fiber optic sensors provide a competitive alternative for ultrasound detection due to the advantages of small size, high sensitivity and excellent immunity to EMI ${ }^{11-15}$. Until now, optical fiber sensors including interferometric

1School of Optical and Electronic Information \& National Engineering Laboratory for Next Generation Internet Access System (NGIA) \& Wuhan National Laboratory for Optoelectronics (WNLO), Huazhong University of Science and Technology, Wuhan 430074, China; ${ }^{2}$ Aston Institute of Photonic Technologies, Aston University, Birmingham B4 7ET, UK.

*Correspondence: QZ Sun, E-mail: qzsun@mail.hust.edu.cn

Received: 2 November 2020; Accepted: 23 December 2020; Published online: 13 April 2022

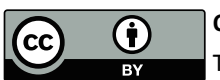

Open Access This article is licensed under a Creative Commons Attribution 4.0 International License.

To view a copy of this license, visit http://creativecommons.org/licenses/by/4.0/.

(C) The Author(s) 2022. Published by Institute of Optics and Electronics, Chinese Academy of Sciences. 
and non-interferometric sensors have been proposed and applied in ultrasound detection. Among these various sensors, Fabry-Pérot interferometer (FPI) ${ }^{12,14,16}$ and Mach-Zehnder interferometer (MZI) ${ }^{17-19}$ are extensively studied and widely utilized in ultrasound detection. High finesse fiber based FPI owns the superiorities of high sensitivity and compact structure. However, the interrogation method based on FPI for ultrasound detection usually requires a tunable laser to track the highest slope in cavity transfer function, which is costly and complicated. Meanwhile, it is hard to fabricate multiple FPIs with identical cavity length, which makes it difficult to realize the multiplexing of FPI ultrasound sensors. Compared with FPI sensors, MZI based ultrasound sensors can be multiplexed in multipoint ultrasound detection, which is cost-effective and time-efficient ${ }^{17,20}$. However, the sensitivity is relatively low due to the large Young's modulus of the silica fiber, which limits its application in weak ultrasound signal detection ${ }^{18}$. Recently, polymer fibers with low Young's modulus were adopted in the MZI to improve the sensitivity of ultrasound detection ${ }^{17}$. While the flawless coupling between polymer fiber and single-mode fiber (SMF) is challenging, which makes the polymer fiber ultrasound sensor difficult to meet the requirement of practical application. Therefore, fiber ultrasound sensors with high sensitivity, ease of fabrication and low cost are still in great demand.

Optical microfiber, with a diameter ranging from hundreds of nanometers to tens of microns, has attracted much attention from researchers ${ }^{21-24}$. Compared with single mode fiber, microfiber offers a smaller size and larger evanescent field, thereby enabling detailed visualization of the realistic ultrasound field with less disturbance. Recently, H. Fan proposed a hybrid structure composed of chalcogenide microfiber and silica microfiber, in which the higher order mode was excited and generated the mode interference ${ }^{25}$. To achieve the highest acoustic sensitivity, it is necessary to lock the interrogation laser wavelength at the point of the highest slope in the interference spectrum of the sensor. However, the combination of chalcogenide microfiber and silica microfiber is not stable due to the weak Van der Waals force, resulting in the interference spectrum susceptible to the disturbance of the external environment, and thus reducing the accuracy of measurement.

In this work, a stable microfiber-based ultrasound sensor with ultra-high sensitivity is proposed. The microfiber is fabricated by tapering a SMF into micrometer size and then packaged by polydimethylsiloxane (PDMS) material. The large evanescent field of microfiber enhances the interaction of light and ambient medium. With the high elastic-optic coefficient of PDMS, the incident ultrasound wave is efficiently converted to the refractive index modulation of PDMS, which further enhances the sensor's sensitivity to ultrasound wave. Meanwhile, the microfiber wrapped in PDMS is in a relatively stable state, which reduces the interference caused by environmental noise. Assisted with the MZI based coherent demodulation system and phase feedback stabilization technology, the proposed sensor achieves a noise equivalent pressure (NEP) as low as $0.15 \mathrm{kPa}$ and exhibits excellent long-term stability. Furthermore, a photoacoustic tomography system is built on the proposed microfiber ultrasound sensor, which realizes human hair imaging with high axial resolution of 65-102 $\mu \mathrm{m}$ and lateral resolutions of $250-420 \mu \mathrm{m}$ over a depth of $12 \mathrm{~mm}$.

\section{Methods and experiment setup}

The fabrication procedure is briefly illustrated in Fig. 1(a). The microfiber used in the work was fabricated by flame-drawing method. Specifically, the SMF was fixed firmly on the motors and then moved the motors slowly until the diameter of microfiber reaches the desired value. Next, the microfiber was carefully transferred onto the PMMA plate. The plate was covered by a thin layer of PDMS (a weight ratio of the base and the curing agent is $10: 1)$ to prevent the microfiber from directly contacting with the PMMA plate. After that, a small amount of PDMS liquid was instilled on the surface of microfiber. The liquid would diffuse slowly and form a uniform protective film. Finally, the PMMA plate was heated at $80^{\circ} \mathrm{C}$ for 20 minutes to solidify the PDMS. In this work, the diameter and length of fabricated microfiber are about 7 $\mu \mathrm{m}$ and $10 \mathrm{~mm}$, respectively, which have both of strong evanescent field and enough strength. Moreover, the proportion of evanescent field transmitted in PDMS is calculated about 2.4\% through COMSOL 5.3. The micrograph of microfiber is shown in Fig. 1(b).

Due to the large evanescent field of the microfiber, the effective refractive index of the microfiber depends on both the refractive index of microfiber and PDMS ${ }^{26}$ :

$$
n_{\mathrm{eff}}=\frac{k_{0}\left[\eta n_{1}^{2}+n_{\mathrm{p}}^{2}(1-\eta)\right]}{\beta},
$$

where $n_{\mathrm{eff}}$ is the effective refractive index of the microfiber, $n_{1}$ and $n_{\mathrm{p}}$ indicate the refractive index of the 


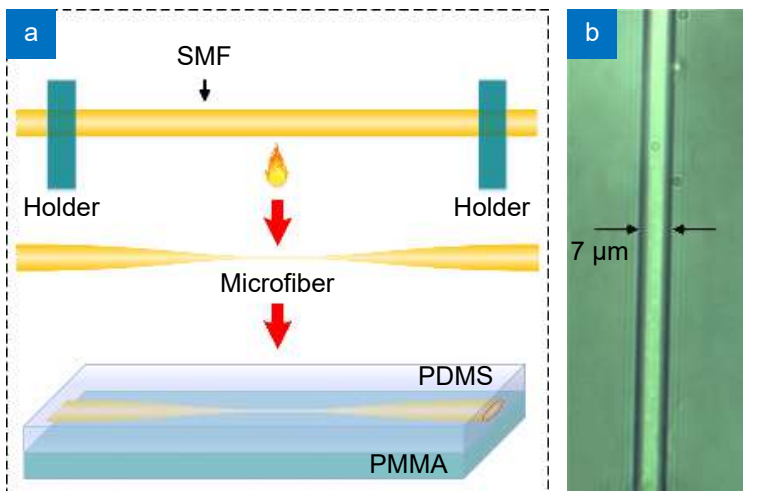

Fig. 1 | (a) Fabrication procedure of the microfiber-based ultrasound sensor. (b) Micrograph of the prepared microfiber.

microfiber and PDMS, respectively. $k_{0}$ denotes the wave number in vacuum, $\eta$ is the proportion of the light energy transmitted in the microfiber and $\beta$ is the propagation constant of the fundamental mode. When the acoustic wave is applied to the microfiber ultrasound sensor, the change of refractive index of the microfiber can be ignored due to the silica microfiber has a very small elastic coefficient. And the refractive index of the PDMS will be modulated owing to the elastic-optic effect, which can be expressed as:

$$
\mathrm{d} n_{\mathrm{p}}=\alpha P_{0},
$$

where $\mathrm{d} n_{\mathrm{p}}$ denotes the change in the refractive index of PDMS, $\alpha$ is the elasto-optic coupling coefficient of PDMS and $P_{0}$ is the applied acoustic pressure. According to the Eq. (1) and (2), the sensitivity of microfiber can be improved by using an encapsulated material with higher elastic-optic coefficient. Moreover, the acoustic impedance of encapsulated material should match the acoustic impedance of water to facilitate acoustic propagation. Compared with water media, PDMS has a higher elastic-optic coefficient and is more efficient in converting acoustic pressure into refractive index change. Since the evanescent field of the microfiber is propagated in the PDMS, the refractive index change of the PDMS will be transmitted to the effective refractive index variation of the microfiber. To interrogate the microfiber-based ultrasound sensor, a beam of interrogation light is injected into the sensor, and then the phase of interrogation light will be modulated by the change of the effective refractive index of the microfiber, which carry the information of the incident acoustic wave. The phase sensitivity can be calculated as follows:

$$
\frac{\mathrm{d} \phi}{P_{0}}=k_{0}\left(n_{\mathrm{eff}} \frac{\mathrm{d} L_{\mathrm{eff}}}{P_{0}}+L_{\mathrm{eff}} \frac{\mathrm{d} n_{\mathrm{eff}}}{P_{0}}\right),
$$

here, $L_{\text {eff }}$ is the effective sensing length of the microfiber sensor, $\mathrm{d} L_{\text {eff }}$ represents the elongation of the effective sensing length and $\mathrm{d} n_{\text {eff }}$ denotes the effective refractive index change of the guided mode. Considering that the length of the microfiber is much longer than the wavelength of the acoustic wave and the sensor is axially constrained, the axial elongation can be ignored. Therefore, the phase modulation is mainly determined by the effective refractive index change.

To test the performance of the fabricated microfiber ultrasound sensor, a MZI with phase feedback mechanism was used for signal demodulation. The interrogating system is exhibited in Fig. 2. A narrow linewidth laser with the operating wavelength of $1550.12 \mathrm{~nm}$ is served as the light source. The incident light is split into reference light and probe light by a $20 \%: 80 \%$ coupler, which are coupled into reference arm and sensing arm, respectively. The microfiber ultrasound sensor is inserted into the sensing arm to modulate the incident ultrasound signal to the phase of probe light. To achieve optimal sensitivity, the MZI has to operate at the working-point, where the phase difference between sensing and reference arm is $\pi / 2$. The working-point can be maintained by an ana$\log$ controller in conjunction with a phase modulator in the reference arm. Then two beams are coupled into a $50 \%: 50 \%$ coupler. The generated interference signal is received and converted to electric signal by a balanced photodetector (BPD), and then the electric signal is split to alternating current (AC) and direct current (DC) signals. The slow shift of the MZI working-point is indicated by the DC signal, which is fed to the analog controller to compensate for the phase fluctuation induced by the temperature variation as well as other low-frequency disturbances. In this way, the MZI will operate at the working-point and the phase variation caused by the ultrasound wave can be linearly translated to the intensity change. A data acquisition card (DAQ) is employed to capture the output AC signal of the BPD and digitize the analog voltage signal. Briefly, the amplitude of the AC signal is proportional to the ultrasound signal pressure. An unfocused piezoelectric transducer (V325, Olympus) and a laser-generated ultrasound source are employed to evaluate the sensitivity and acoustic frequency response of the sensor, respectively. The sensor is placed in front of the acoustic sources and perpendicular to the direction of the ultrasound propagation. The measured results are compared with a commercial ultrasound hydrophone (NH200, Precision Acoustics) for calibration. 


\section{Results and discussion}

To evaluate the sensitivity of the microfiber ultrasound sensor, a $2.25 \mathrm{MHz}$ unfocused ultrasound transducer was used as the ultrasound source. The pressure of generated ultrasound signal was $55 \mathrm{kPa}$, which was calibrated by the commercial hydrophone. The typical measured signal is shown in Fig. 3(a), the peak-to-peak output voltage is $190 \mathrm{mV}$, and thus the sensitivity of the microfiber ultrasound sensor is estimated as $3.5 \mathrm{mV} / \mathrm{kPa}$. As shown in Fig. 3(a), the acquired data within $1 \mu$ s before the ultrasound signal arrived is used to assess the noise level, the root-mean-square noise level is about $0.54 \mathrm{mV}$ over a 20 $\mathrm{MHz}$ measurement bandwidth and the signal-to-noise (SNR) can be calculated as $51 \mathrm{~dB}$. Therefore, the corresponding noise equivalent pressure (NEP) is estimated to be $0.15 \mathrm{kPa}\left(34 \mathrm{mPa} \cdot \mathrm{Hz}^{-1 / 2}\right)$. Compared with the polyvinylidene fluoride (PVDF) ultrasound sensor ${ }^{27}$, which has a diameter of $200 \mu \mathrm{m}$ and a NEP of $3 \mathrm{kPa}$, the microfiber ultrasound sensor exhibits a 20 times improvement in NEP with a diameter of only $7 \mu \mathrm{m}$.
Moreover, the NEP can be further reduced by using a microfiber with strong evanescent field. The voltages of output signals with different pressures are also collected and exhibited in Fig. 3(b). It can be seen that in the range of 0 to $550 \mathrm{kPa}$, the acoustic pressure and output voltage shows a good linearity with R-square of 0.99 . The fitting curve for the sensitivity of the microfiber ultrasound sensor has an intercept, which is caused by the sensitivity fluctuation of microfiber sensor and the measurement uncertainty of the calibration hydrophone. To fully reveal the sensitivity enhancement of microfiber, a contrast experiment based on SMF sensor was also conducted. It can be seen that the sensitivity of the SMF sensor is only about $0.39 \mathrm{mV} / \mathrm{kPa}$, which is approximately 10 times lower than the proposed microfiber-based sensor. From the comparison, the proposed microfiber ultrasound sensor is more suitable for the detection of weak signals.

For capturing faithful ultrasound wave, the sensor should possess a broad bandwidth covering from hundreds of $\mathrm{kHz}$ to tens of $\mathrm{MHz}$. In order to evaluate the

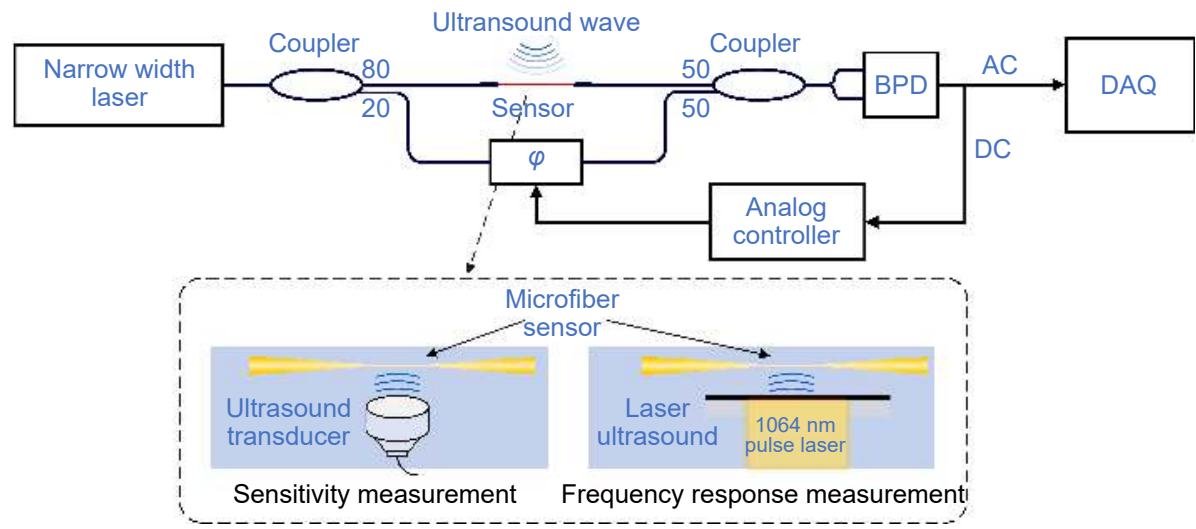

Fig. 2 | The diagram of ultrasound detection system. $\varphi$ : Phase modulator. BPD: Balanced photodetector. DC: Direct current signal. AC: Alternating current signal. DAQ: Data acquisition card.
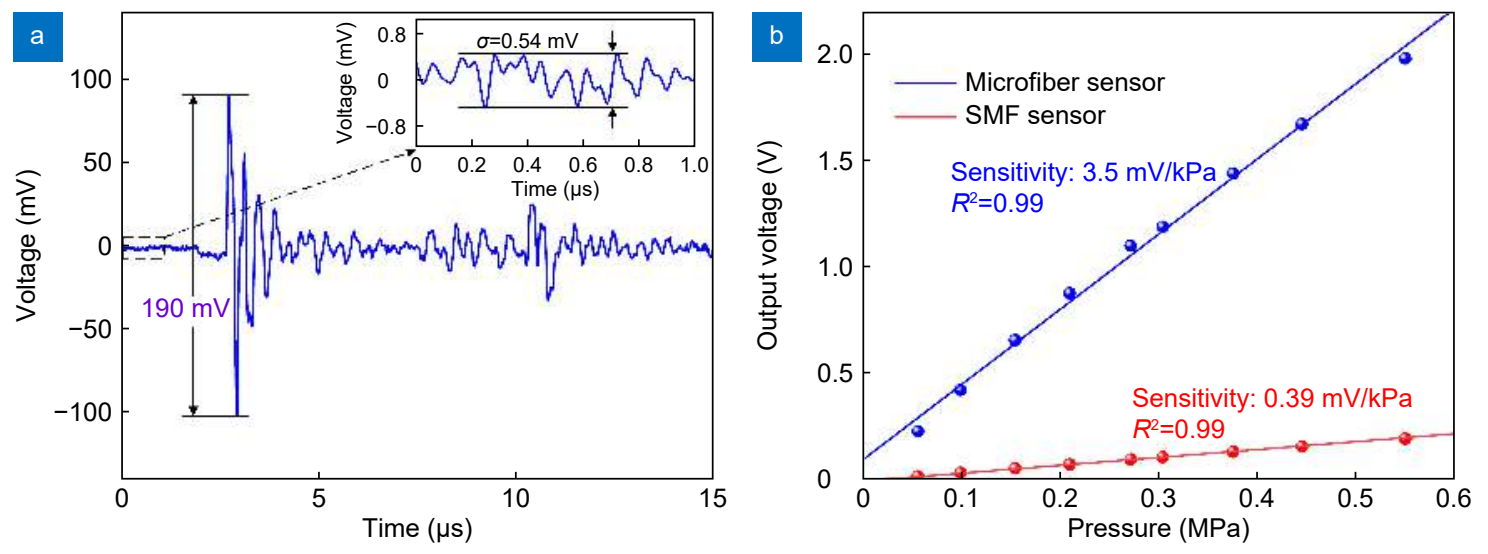

Fig. 3 | (a) Time response of the sensor to an ultrasound pulse. (b) Ultrasound pressure sensitivities of microfiber sensor and SMF sensor. 
frequency response of the microfiber sensor, a broad bandwidth acoustic source based on photoacoustic effect $^{28}$ was used. The acoustic source was created by illuminating the black paint coating using a 6-ns pulsed laser with a spot size of $5 \mathrm{~mm}$ in diameter. Fig. 4(a) and 4(b) depict the recorded response to acoustic pulse in time and frequency domains, respectively. It can be observed that the temporal waveform is a clear monopolar signal free from ringing. It should be noted that the peak $A$ is the original acoustic pulse and the adjacent peak B is generated by the reflection of the encapsulated PMMA plate, which can be eliminated by using acoustic impedance matching materials. Moreover, the broadband frequency response of the microfiber sensor extended to approximately $40 \mathrm{MHz}$ and the $-10 \mathrm{~dB}$ bandwidth is 14 MHz. The excellent indicators of the proposed sensor can meet the requirements of high-resolution endoscopic ultrasound and photoacoustic imaging.

Moreover, the short-term and long-term stability of the sensor were verified as well. The sensitivity was con-

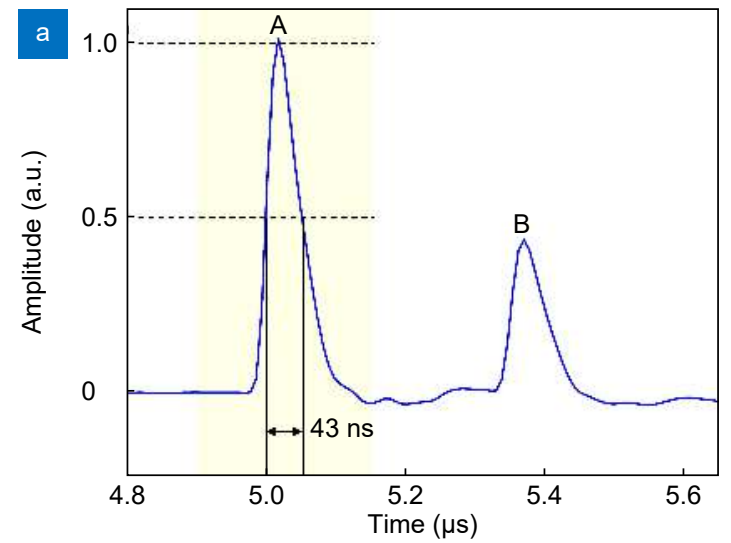

Fig. 4 | (a) Recorded signals in response to broadband ultrasound wave. sensor. tinuously recorded as long as 110 hours with a time interval of 24 hours. The experimental method was the same as the approach used in sensitivity measurement. For each test, the data was recorded for 10 times to reduce the measurement error and evaluate short-term fluctuations. As shown in Fig. 5, the maximum fluctuation of the sensitivity within 110 hours is less than $\pm 8 \%$ and the maximum error bar of each test is $1.2 \%$. The dominant factors that may lead to variation of sensitivity are discussed as follows. On the one hand, when the sensor or demodulation system is subjected to rapid vibration, while the phase feedback system cannot follow the state in time due to the response time, resulting in the deviation from the working-point of the MZI. On the other hand, the inhomogeneity of optical fibers will cause the slow polarization change during the light transmission, and further result in the polarization fading, which can be eliminated by employing the polarizationmaintaining fiber.

Benefiting from high sensitivity, broadband frequency

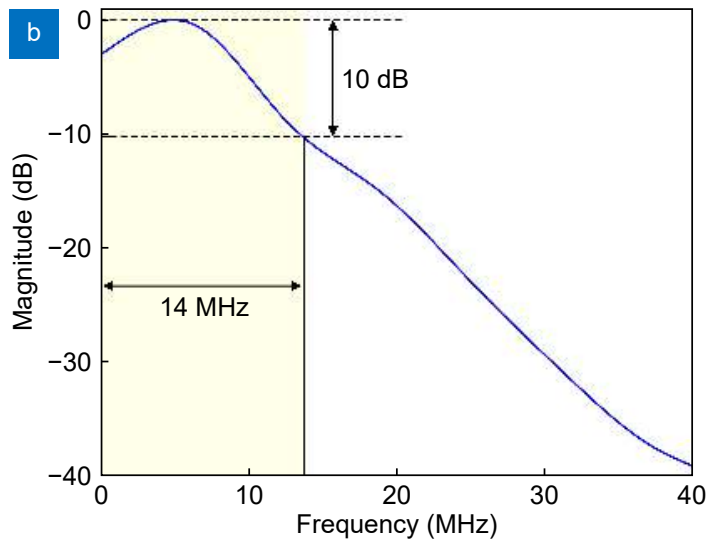

(b) Measured frequency responses of the microfiber-based ultrasound

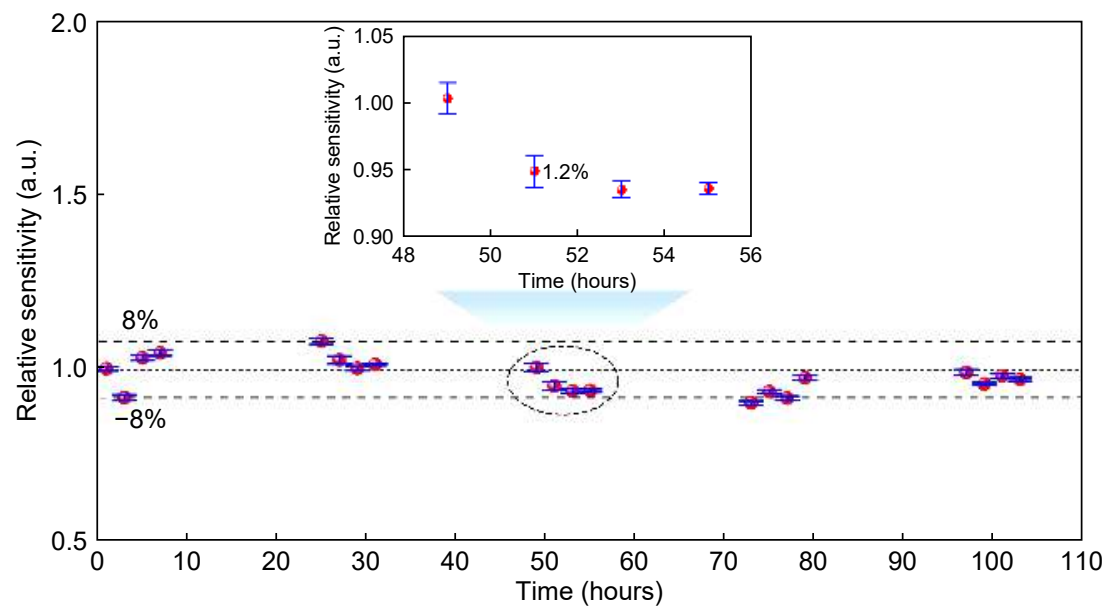

Fig. 5 | The short-term and long-term stability of the microfiber-based ultrasound sensor. 
response and good stability, the proposed sensor was further utilized for photoacoustic tomography. The diagram of the imaging system is presented in Fig. 6. A 1064 nm Nd:YAG pulsed laser (DAWA-100, Beamtech) with a fixed pulse duration of $8 \mathrm{~ns}$ is served as the excitation

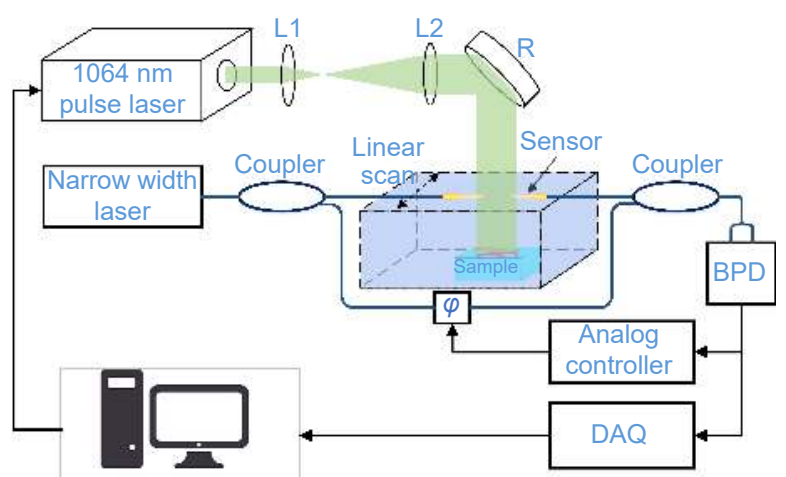

Fig. 6 |Schematic of the photoacoustic tomography imaging system. L1\&L2: Lens. R: Reflector.
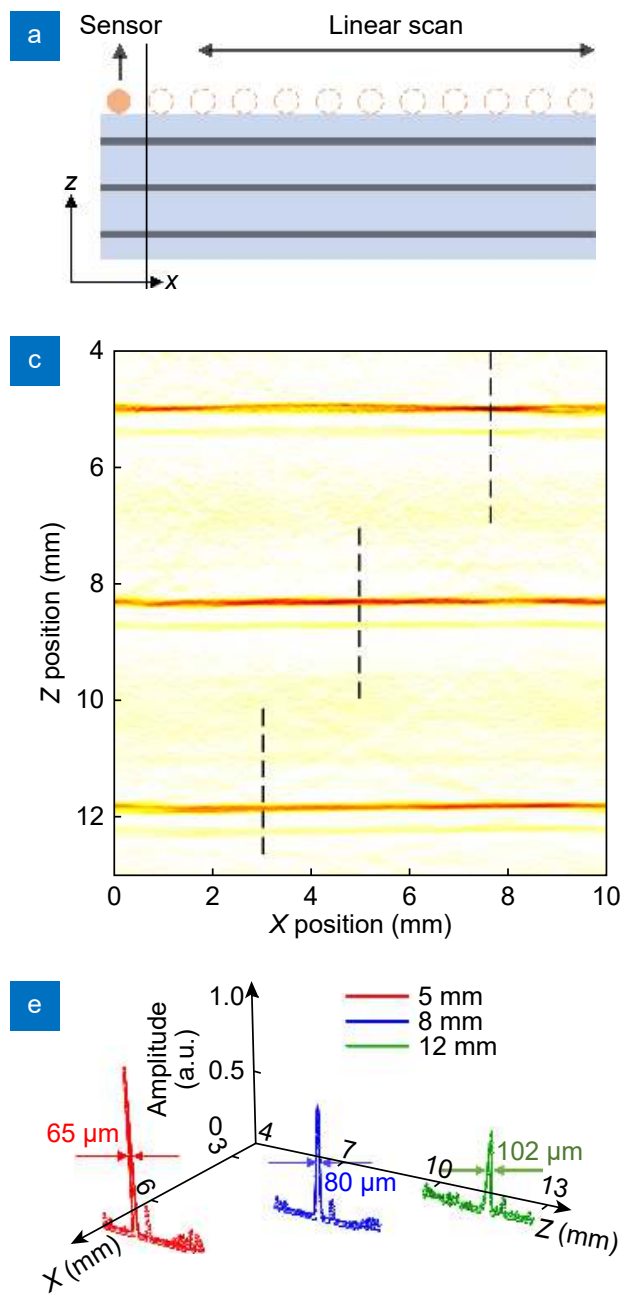

light source. A set of mirrors and lenses are used to expand the laser beam to a diameter of $25 \mathrm{~mm}$. The laser works at a $10 \mathrm{~Hz}$ repetition rate with the single-pulse energy of $80 \mathrm{~mJ}$ and the optical fluence on the sample surface is $16 \mathrm{~mJ} / \mathrm{cm}^{2}$, which is below the American National Standards Institue (ANSI) safety limit. Assisted by a motor, the fabricated sensor achieves the detection of the target at a scanning step of $50 \mu \mathrm{m}$. The measurement signals are digitalized by a DAQ at a sampling rate of 125 $\mathrm{MHz}$. The excitation laser, motor and DAQ are controlled and synchronized by a LabVIEW program. After data acquisition, a time-reversal algorithm is employed to reconstruct the photoacoustic images ${ }^{29}$.

To characterize the lateral and vertical spatial resolution of the microfiber-based photoacoustic tomography system, the method of imaging small samples is usually adopted $^{30}$. Therefore, three parallel hairs with a diameter
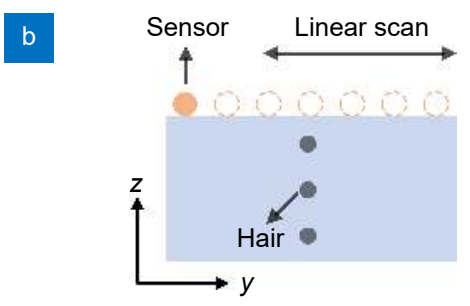

d
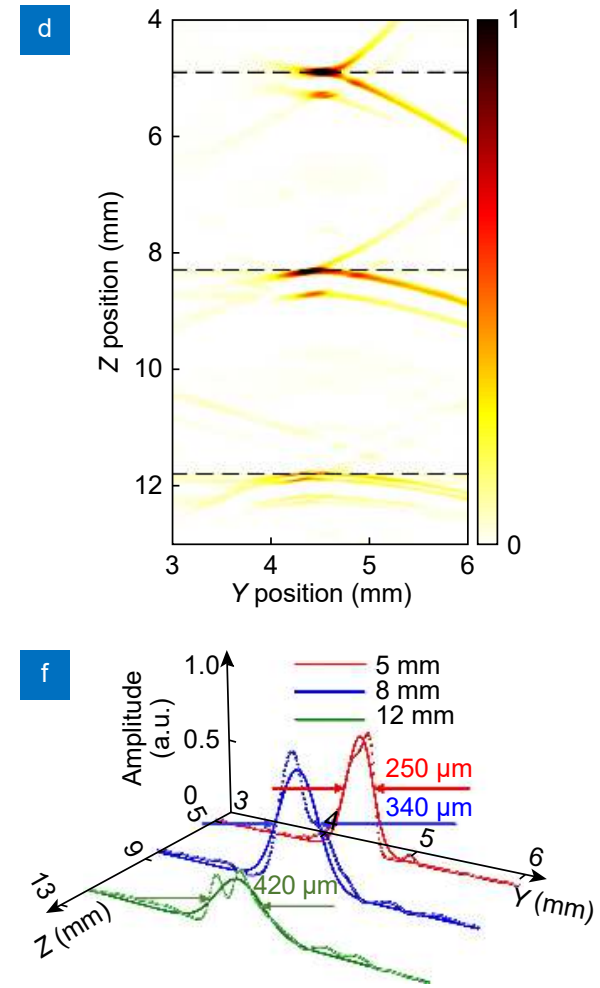

Fig. 7 | Images of three human hairs using the photoacoustic tomography system. (a, b) The sensor is placed perpendicular and parallel to hairs in the axial and lateral resolution measurements, respectively. (c, d) The reconstructed images of the hairs corresponding to (a) and (b), respectively. (e, f) The axial and lateral resolutions at different imaging depths. 
of $60 \mu \mathrm{m}$ were used as the samples, which were placed at depths of $5 \mathrm{~mm}, 8 \mathrm{~mm}$, and $12 \mathrm{~mm}$, respectively. As shown in Fig. 7(a) and 7(b), the microfiber sensor is perpendicular and parallel to the hairs respectively in axial and lateral resolution measurements. The corresponding reconstructed images of the sample are exhibited in Fig. 7 (c) and 7(d), respectively. Three hairs can be clearly observed in images while the shadow below each hair is caused by the reflected signal of PMMA substrate. The data along the dotted lines in Fig. 7(c) and 7(d) are extracted and fitted by Gaussian curves, of which the resolution is determined by the full width at half maximum. From Fig. 7(e) and 7(f), the axial spatial resolution gradually deteriorated from $65 \mu \mathrm{m}$ to $102 \mu \mathrm{m}$ as the imaging depth increased from $5 \mathrm{~mm}$ to $12 \mathrm{~mm}$. The lateral spatial resolution is $250 \mu \mathrm{m}$ at the depth of $5 \mathrm{~mm}$ and gradually increased to $420 \mu \mathrm{m}$ at the depth of $12 \mathrm{~mm}$. The decrease in axial resolution is mainly attributed to the fact that the transmission loss of higher frequency acoustic wave is relatively high. While owing that the effective angular detection aperture decreases along with the increasing depth, the lateral resolution becomes larger resulting in the fuzzy imaging. Notably, the amplitudes of reconstructed image decrease with the increase of depth, due to the large transmission attenuation of the ultrasound wave.

\section{Conclusions}

In summary, this work demonstrated a microfiber-based ultrasound sensor as well as an MZI interrogating system to achieve high sensitivity and stable ultrasound detection. Due to the large evanescent field characteristics of microfiber and the high elastic-optic coefficient of PDMS, the microfiber-based ultrasound sensor exhibits a sensitivity as high as $3.5 \mathrm{mV} / \mathrm{kPa}$, which is approximately 10 times higher than the SMF based sensor. The sensor also exhibits a low NEP of $0.15 \mathrm{kPa}$ and large dynamic range from 0 to $550 \mathrm{kPa}$. Moreover, benefiting from the phase feedback stabilization technology and microfiber encapsulation with PDMS, the excellent stability of the system is demonstrated, with the maximum fluctuation of sensitivity less than $\pm 8 \%$ within 110 hours. Furthermore, a PAT system based on the microfiber ultrasound sensor is established, which achieves an axial resolution of $65-102 \mu \mathrm{m}$ and lateral resolution of $250-420 \mu \mathrm{m}$ over a depth of $12 \mathrm{~mm}$. The sensor shows great potential for NDT and biomedical ultrasound imaging.

\section{References}

1. Li C, Wang LV. Photoacoustic tomography and sensing in biomedicine. Phys Med Biol 54, R59-97 (2009).

2. Beard P. Biomedical photoacoustic imaging. Interface Focus 1, 602-631 (2011).

3. Powers J, Kremkau F. Medical ultrasound systems. Interface focus 1, 477-489 (2011).

4. Le Jeune L, Robert S, Villaverde EL, Prada C. Plane Wave Imaging for ultrasonic non-destructive testing: Generalization to multimodal imaging. Ultrasonics 64, 128-138 (2016).

5. Drinkwater BW, Wilcox PD. Ultrasonic arrays for non-destructive evaluation: A review. NDT E Int 39, 525-541 (2006).

6. Hekmati A, Hekmati R. Optimum acoustic sensor placement for partial discharge allocation in transformers. IET Sci Meas Technol 11, 581-589 (2017).

7. Sarkar B, Mishra DK, Koley C, Roy NK, Biswas P. IntensityModulated Fiber Bragg Grating Sensor for Detection of Partial Discharges Inside High-Voltage Apparatus. IEEE Sens J 16, 7950-7957 (2016).

8. Janapati V, Kopsaftopoulos F, Li F, Lee SJ, Chang FK. Damage detection sensitivity characterization of acousto-ultrasoundbased structural health monitoring techniques. Structural Health Monitoring-an International Journal 15, 143-161 (2016).

9. Qiu YQ, Gigliotti JV, Wallace M, Griggio F, Demore CEM et al. Piezoelectric Micromachined Ultrasound Transducer (PMUT) Arrays for Integrated Sensing, Actuation and Imaging. Sensors 15, 8020-8041 (2015).

10. Xia WF, Piras D, van Hespen JCG, van Veldhoven S, Prins $C$ et al. An optimized ultrasound detector for photoacoustic breast tomography. Med Phys 40, 13 (2013).

11. Rosenthal A, Razansky D, Ntziachristos V. High-sensitivity compact ultrasonic detector based on a pi-phase-shifted fiber Bragg grating. Opt Lett 36, 1833-1835 (2011).

12. Guggenheim JA, Li J, Allen TJ, Colchester RJ, Noimark $S$ et al. Ultrasensitive plano-concave optical microresonators for ultrasound sensing. Nat Photonics 11, 714-719 (2017).

13. Ma XD, Cai YQ, Fu B, Xu LJ, Ma JG. Fiber optic-based laser interferometry array for three-dimensional ultrasound sensing. Opt Lett 44, 5852-5855 (2019).

14. Wang $X X$, Jiang YH, Li ZY, Wang W, Li ZB. Sensitivity Characteristics of Microfiber Fabry-Perot Interferometric Photoacoustic Sensors. J Lightwave Technol 37, 4229-4235 (2019).

15. Bai $X, Q i Y$, Liang $Y$, Ma J, Jin $L$ et al. Photoacoustic computed tomography with lens-free focused fiber-laser ultrasound sensor. Biomedical Optics Express 10, 2504-2512 (2019).

16. Fan HB, Zhang L, Gao S, Chen L, Bao XY. Ultrasound sensing based on an in-fiber dual-cavity Fabry-Perot interferometer. Opt Lett 44, 3606-3609 (2019).

17. Bauer-Marschallinger J, Felbermayer K, Berer T. All-optical photoacoustic projection imaging. Biomed Opt Express 8, 3938-3951 (2017).

18. Nuster R, Gratt S, Passler K, Grun H, Berer T et al. Comparison of optical and piezoelectric integrating line detectors. Photons Plus Ultrasound: Imaging and Sensing 2009, edn, 7177, (Spie-Int Soc Optical Engineering, Bellingham, 2009).

19. Lamela H, Gallego D, Oraevsky A. Optoacoustic imaging using fiber-optic interferometric sensors. Opt Lett 34, 3695-3697 (2009).

20. Bauer-Marschallinger J, Höllinger A, Jakoby B, Burgholzer P, 
Berer T. Fiber-optic annular detector array for large depth of field photoacoustic macroscopy. Photoacoustics 5, 1-9 (2017).

21. Zhang L, Pan J, Zhang Z, Wu H, Yao N et al. Ultrasensitive skin-like wearable optical sensors based on glass micro/nanofibers. Opto-Electronic Adv 3, 190022 (2020).

22. Tong L, Gattass RR, Ashcom JB, He S, Lou J et al. Subwavelength-diameter silica wires for low-loss optical wave guiding. Nature 426, 816-819 (2003).

23. Zhang Z, Pan J, Tang $Y, X u Y$, Zhang $L$ et al. Optical micro/nanofibre embedded soft film enables multifunctional flow sensing in microfluidic chips. Lab on a Chip 20, 2572-2579 (2020).

24. Zhang NMY, Li KW, Zhang N, Zheng Y, Zhang T et al. Highly sensitive gas refractometers based on optical microfiber modal interferometers operating at dispersion turning point. Optics Express 26, 29148-29158 (2018).

25. Fan $H$, Chen $L$, Bao X. Chalcogenide microfiber-assisted silica microfiber for ultrasound detection. Opt Lett 45, 1128-1131 (2020).

26. Stewart G. Optical waveguide theory. (Chapman and Hall, 1983).

27. Beard PC, Hurrell AM, Mills TN. Characterization of a polymer film optical fiber hydrophone for use in the range 1 to $20 \mathrm{MHz}$ : A comparison with PVDF needle and membrane hydrophones. leee Transactions on Ultrasonics Ferroelectrics and Frequency Control 47, 256-264 (2000).

28. Lu QB, Liu T, Ding L, Lu MH, Zhu J et al. Probing the Spatial
Impulse Response of Ultrahigh-Frequency Ultrasonic Transducers with Photoacoustic Waves. Physical Review Applied 14, 034026 (2020).

29. Treeby BE, Cox BT. k-Wave: MATLAB toolbox for the simulation and reconstruction of photoacoustic wave fields. Journal of Biomedical Optics 15, 021314 (2010).

30. Li G, Guo Z, Chen SL. Miniature all-optical probe for large synthetic aperture photoacoustic-ultrasound imaging. Optics Express 25, 25023-25035 (2017).

\section{Acknowledgements}

We are grateful for financial supports from National Natural Science Foundation of China (NSFC) (No. 61922033); Foundation for Innovative Research Groups of Hubei Province of China (2018CFA004); and Innovation Fund of WNLO.

\section{Author contributions}

L. Y. Yang and Q. Z. Sun proposed the original idea the project. Q. Z. Sun supervised the project. L. Y. Yang, Y. P. Li, and L. Y. Li participated the discussion of the research. L. Y. Yang and F. Fang carried out the experiments and collected the data. L. Y. Yang analysed all the data. L. Y. Yang and Q. Z. Sun wrote the paper. All authors discussed the results and commented on the manuscript.

\section{Competing interests}

The authors declare no competing financial interests. 